\title{
Critical Analysis of Complex Systems Science and Mechanics
}

\author{
Shi Xiao Qin \\ ${ }^{1}$ School of Materials Science and Engineering, Sun Yat-sen University, Xingang Xi Road, P. R. China. \\ 19insunyat2045@gmail.com
}

\begin{abstract}
ArticleInfo
Journal of Machine and Computing (http://anapub.co.ke/journals/jmc/jmc.html)

Doi : https://doi.org/10.53759/7669/jmc202101023
\end{abstract}

Received 30 March 2021; Revised form 30 June 2021; Accepted 18 August 2021; Available online 05 October 2021.

(O2021 The Authors. Published by AnaPub Publications.

This is an open access article under the CC BY-NC-ND license. (http://creativecommons.org/licenses/by-nc-nd/4.0/)

\begin{abstract}
For many highly complex, ecological, physical and societal structures, the conventional hypotheses that underpin many theoretical and analytical frameworks are false. Complex systems research elucidates why and when such preconceptions are incorrect, as well as alternate paradigms for comprehending complex series characteristics. Complexity characteristics, the tradeoff between effectiveness and adaptation, the need of matching the complexities of networks to that of their surroundings, multiresolution assessment, and evolution are among the fundamental concepts of Complex Systems (CS) research introduced in this study. Instead of simulating particular dynamics, we concentrate on the general characteristics of systems. We didactically explain a theoretical and analytic strategy for comprehending and engaging with the complicated processes of our environment rather than giving a complete overview. This article requires just a middle school mathematics and science foundation in order to make it approachable to researchers from all disciplines, decision-makers from business, government, and charity, and anybody engaged in networks and civilization.
\end{abstract}

Keywords - Complex Systems Science (CSSs), Complex Systems (CS), Machines, Critical Systems.

\section{INTRODUCTION}

How can we investigate complex structures, physiological, and sociological systems - in a rational manner? While investigations are valuable, they are inadequate in and of itself since all tests need a conceptual base to understand them. While there are numerous paradigms for studying individual elements or features of structures, the basic assumptions that underpin most quantitative approach frequently do not apply to structures as a whole, leading in a misinterpretation of the ramifications of large-scale behaviors. This article gives an overview of system dynamics engineering and shows how it may be used to make better choices in our world's dynamic structures. It emphasizes on certain generic characteristics of complex processes rather than simulating particular dynamics like the subdisciplines of stochastic processes, agent-based modelling, and evolutionary computation, social network analysis, and quantum physics do. The core concepts of Complex Systems Sciences (CSSs) are the subject of Section II. Section III focuses on Complex Structures (Cs) and Uncertainties. Finally, Section IV concludes the research.

\section{BASIC PRINCIPLES}

Reasons for the Complex Systems Sciences (CSSs)

Complex systems science (CSSs) looks at structures with a lot of moving parts. Geological, psychological, or social dynamics may be involved. Considering the range of programs, it may appear odd to examine them all under the same paradigm [1]. CSSs, on the other hand, concentrate about how the elements within structures are linked to each other, whereas most biological sciences prefer to concentrate on the elements individually. Complex adaptive research, for example, organizes the subsystems in Figure 1 by rows, whereas most scholarly fields arrange them by columns.

Every column involves 3 illustrations of systems made up of the same elements (compounds, tissues, and humans, spanning left to right), however with various relationships among them. Every row includes systems that depict a particular type of component-to-component interaction. Every element's activity in a random system is autonomous of other elements' behaviors. Each element of a coherent structure behaves in the same way. The response of one component of a projectile, for instance, fully influences the activity of the other components (position, direction, and speed). Correlated systems are in the middle, where the responses of the system 's elements are interrelated but not fully determined by one another; for example, the form of one portion of a snowflakes is associated but not entirely determined by the structure of all the other elements. The need to define the collection of actions under investigation is implicit in these representations.

Structures may vary from one another not by variations in their components, but rather because of variances in how components interact with and influence each other [2]. Heat and snow, for instance, are made up of the same hydrogen atoms but have vastly different characteristics owing to changes in the bonds amongst them. In contrast, despite variations in their component molecules, all gases have many characteristics in common. Liquids and solids behave the same way. The characteristics that differentiate minerals from liquids and gases are instances of onset: they cannot be predicted from the components of a system alone. 

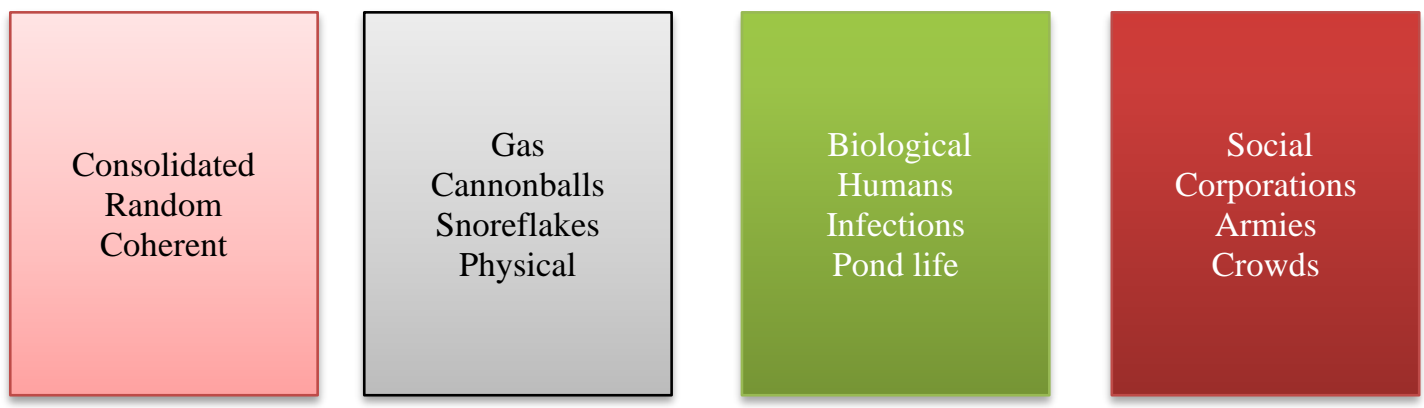

Fig 1. Components of complex adaptive research for coherent networks classified in rows

Fluid instability, as seen in a moving river, is an illustration of how parts' interactions may lead to self-organized humongous behaviours and structures. The creation of discussion groups at a party, the distribution of commodities in a decentralised market, the development of environments, and the flocking of birds are all instances of self-organized behaviours. These large-scale behaviours and tendencies are impossible to predict by looking at each system component separately. Complex systems science offers a multidisciplinary research paradigm for the development of new knowledge, technologies, and linkages by focusing on general characteristics of systems in totality rather than individual components [3].

Even for relatively basic systems, it is difficult to fully describe all of the small-scale features. As a result, sound assessments must only explain system characteristics that are unaffected by all of these details. Universality is responsible for the existence of such characteristics. Whilst describing the performance of an individual state of the process (- for example, a vapor) could be completely insoluble, summarizing the set of potential dynamical system would not only be feasible but it may also provide a more model of the pertinent data, according to numerical methods (e.g., the pressure, temperature, density, and compressibility). To put it another way, stepping back and examining the universe of potential actions offers a strong analytical lens could be used to biological and cultural systems as well as physical ones.

\section{Definition of "Complexity"}

The breadth of a behavior's descriptions is used to calculate its complexity. The amount of potential behaviours systems may show represents the size of a characterization of its behavior. A light bulb, for example, may be limited to a single piece: Zero or one. 3 items may represent four distinct responses $(0,1,10 / 11), 3$ bits can represent 8 , etc. We can compose $\mathrm{C}=\log 2, \mathrm{~N}$, where $\mathrm{C}$ is the program's complex nature " $\mathrm{N}$ " and is the variety of available behavior patterns (technologically, $\log 2, \mathrm{~N}$ is indeed a theoretical limit for the program's uncertainty because if some behaviour patterns are much more probable over others, the average duration of the program's characterization could be lowered through using shortened explanations for more basic qualities and lengthier characterizations for the less popular options flexural algorithm depends upon the rationality), however for our uses, it is applicable to state that the more the amount of potential behaviours, the more the complexity level.

It's essential to keep in mind that the range of potential actions must be clearly defined. For instance, if we are involved in the uncertainty of constructing an incandescent bulb, the interior of potential behavioural patterns may include many of the infinite permutations of its components; nevertheless, if we are engaged in the uncertainty of constructing an incandescent bulb, the interior of possible behavioural patterns may have all of the infinite permutations of its components, as explained above. Consider training a system to properly answer a four-choice multiple-choice inquiry as another illustration. At first sight, this job seems to be fairly straightforward: there are only two bits needed because there are four potential actions [4]. Nevertheless, we get the impression that training a computer to master a multiple-choice exam would be a tough task. This seeming contradiction is addressed, nevertheless, when we realize that such a work is tough just because we do not even know what items will be on the exam ahead of time, and that the actual goal is to be able to properly answer any multiple-choice questions. Given the huge number of potential responses the computer might make to a series of random numerous questions, this job is extremely difficult.

\section{Scale and Complexity}

Consider a person, and then imagine a gas that has the same elements as the individual and in no specified sequence. Which methodology is more difficult to understand? The gas has a larger number of potential molecular configurations (i.e., it has more unpredictability, or disturbance), thus it would take much longer to explain at the molecular scale. When we conceive of a dynamic network, nevertheless, we conceive of the behaviors that arise from the ordered organization of components in a person, not from the maximum disorderly organization of particles in a gas. As a result, it may be tempting to assume that sophisticated systems have less chaos. However, the least disordered systems are those where all elements behave in the same way (cohesive networks in Fig 1), and such behaviour is simple to explain and therefore not obviously complicated.

To reconcile this seeming contradiction, remember that the complexity of a program's representation is determined by the degree of information required to describe it. As a result, intricacy is size dependent. It's far more difficult to explain 
the locations and speeds of all the particles in a gas on a tiny scale than just doing the same with all the particles in the body. Nevertheless, a gas's activities are guided by its pressures and temperatures on a human scale, while human behavior remains very complicated. The lowest scale of intricacy is entropy, yet describing a system necessitates a knowledge of its complexity at many scales. The intricacy profiles of a systems is a graph representing the system's intricacy as a proportion of scale. The dimension of a behavior will be assumed to be lengths in the cases below, although it is essentially proportionate to the amount of synchronized elements participating in the activity, for which actual measurement is a placeholder. At the level of human vision, a gas is extremely basic because only behaviours comprising billions of particles are significant, and a gas with so many molecules has very few identifiable behaviors.

Randomized, harmonic, and coupled structures (see Fig 1) exhibit fundamentally distinct complexity profiles, as illustrated in Fig 2. Random structures have the greatest intricacy at the microscale (best metadata precision), but when the size is raised and the randomized behaviours of the individual elements are averaging out, the degree of intricacy quickly decreases. Because expressing the overall performance of the network (e.g., the location and speed of a projectile) also explains the behaviour of all the elements, a consistent structure has the same complexities at microscale as it is at bigger ones (such as the velocity and position of various atoms) [5]. Because looking at a systems in more depth (while still include the whole network in the analysis) yields more data, complexity tends to grow (or stay the same) as the scope lowers.

Because different activities occur at multiple scales in a linked system, the intricacy progressively rises as the system is examined in more depth. For example, a person, which is scarcely apparent from afar, has very little intricacy. As the degree of detail increases, the description will begin with the person's general positions and velocities, then the locations and speeds of each arm, then the movements of arms, fingernails, facial gestures, and any words the human may be speaking. As the degree of detail increases, the functions, organs, and rhythms of the human mind become more important as well as the single cells. Vesicles (cell substructures) [6], followed by big compounds like proteins and Nucleic acids, and finally smaller fragments and solitary atoms, add to the intricacy at inches shorter than for a cell. The duration of the explanation increases with each level. A distinguishing feature of complex structures is this amazing multiscale architecture with progressively increasing complexities.

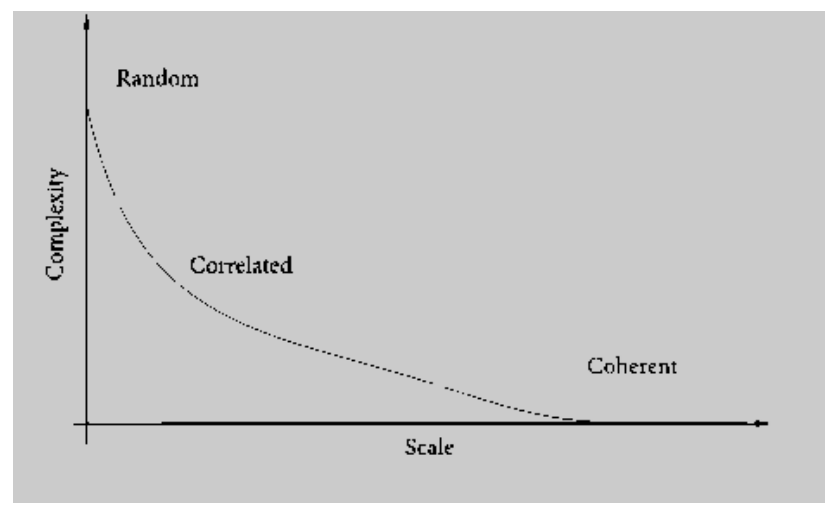

Fig 2. Representative complexities profile for correlated coherent and random structures. At a particular structure might compose various aspects of each in different scales.

\section{Complexity vs. Scale Constraints}

The notion that complex structures is indeed not completely baseless: in order for higher scale behaviours to exist, numerous smaller-scale elements must be coordinated. Since the lower-scale components' actions are now constrained by their interrelations, this cooperation reduces complexity at smaller sizes. The tension among large-scale and smallscale complexity may be quantified in this way: given a defined collection of items and a certain number of possible behavioral aspects, the region underneath the complexity profiles will remain constant irrespective of constituent interrelatedness (or lack hereof). More specifically, the total of a system's intricacy at every level (i.e., the area underneath its complication profile) equals the sum of the intricacy of the each individual part. As a result, there is a basic tradeoff for every system between the amount of behaviors it may have and their size.

Consider a large plant with a large number of employees. A complexity profile (Fig 3) may be used to describe the factory's output. The number of various kinds of products that a factory can make at a particular scale is a metric for that scale's complexity, whereas the number of units of the same category of goods that a manufacturer can manufacture in a given period of time is a reference for scale. The basic tradeoff is that if the firm wishes to produce a large number of copies of a particular kind of product in a short period, it would have to synchronize all of its employees (possibly by putting them on a production line), limiting their individual liberty to produce a variety of commodities [7]. The factory's manufacturing would therefore be simple but large-scale (for example, cranking out numerous identical Model-T Fords"Whatever client may have a vehicle repainted any color he likes as long as this is dark").

If the factory's workers work individually, they would be able to produce a wide range of goods, but nothing at scale. In general, by incorporating more equipment or more employees, a factory may enhance both the intricacy and size of its output; the exact balance amongst scale and complexity holds only when a given set of elements with a given set of 
discrete behaviors is considered. In certain instances, adding contact between different system components may expand the set of important specific behaviors of every part, thus raising the overall area underneath the intricacy profiles. If two individuals communicate with one another, for instance, the interaction ( - for example, communication) may be a crucial behaviour of each member that was not present previously.

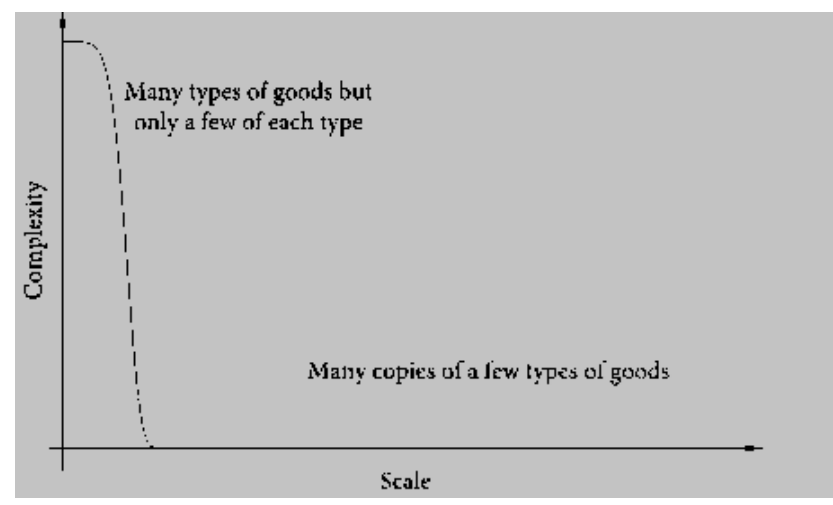

Fig 3. A complexity profile factory's output

The complexity profiles of a firm that can create a huge multiple copies of a few different kinds of products, and the complex pattern of a firm that can manufacture an array of items but not in big quantities. Because commercial manufacturing, based on a fixed system, necessitates larger-scale synchronized industrial activity, the number of units of a product unit is a proxy for size (- for instance, an assembly line). The variety of possible kinds of products that may be manufactured at a particular scale is a metric for the factory's number of varied potential behaviors-and therefore its variability that size.

The trade-offs amongst responsiveness and productivity is a consequence of the complexity vs. scale dilemma. If there are numerous potential activities taking place in simultaneously that are relatively independent of one another, i.e. when the process is complex, resilience emerges. On the contrary, effectiveness occurs when several elements of a system operate together to accomplish the task in which it was constructed at the maximal scale. A system with more adaptation will have a complexities structure with higher complexity but primarily at smaller sizes, while a systems with more effectiveness would have a diversity profiles with lower computational complexity but spreading to massive scales because of the tradeoffs between scope and size [8]. As a result, a highly efficient systems will be less flexible to unexpected changes within itself or in its surroundings owing to its reduced computational complexity, while a highly adaptable systems built to withstand all types of shocks would have to compromise certain larger-scale behaviour.

The Soviets felt they might have their share and consume it, as well: they thought their system would exceed capitalism economies since economic systems had so much waste from many companies vying for the same job. Coordination of all economic output would have been much more effective. However, lower-scale complexity was lost in the process of building such significant economic frameworks, culminating in a deterministic ecosystem. Inadequately administered capitalist economies may also compromise redundancies and flexibility in the name of expediency, culminating in monopolistic tendencies imbalances, negative feedback cycles, and herd-like behaviors. Because of the tradeoff amongst size and complexity, every system that increases larger-scale intricacies market, governmental, or otherwise-must decrease individual intricacy. This isn't to imply that larger-scale intricacy is always bad; sometimes it can be worthwhile it to give up some civil freedoms in exchange for larger-scale collaboration. So, when is it beneficial to have intricacy at a certain scale?

\section{Reasons for Being Complex}

The Principle of Requisite Variation determines when intricacy is preferable: a system should be at minimum as intricate as the ambient responses with which it should selectively respond in order to be successful. If a systems are required to respond differently to each one of a hundred significant ecological scenarios but only has Ten responses available, the system will be ineffective. At a minimum, the program would require a hundred various behaviors, one for each conceivable situation (the preceding criterion is required but not adequate; a systems with enough actions may still fail to execute the appropriate actions in the appropriate conditions). It is worth noting that the surroundings in which systems should respond is a network in and of itself, and would be alluded to as such on occasion.

We may modify the Law of Requisite Variety by stating that for a system to be successful, it must equal (or surpass) the intricacy of the contextual responses with which it should selectively respond at all dimensions where these activities occur. Consider armed warfare as an example of this multiresolution form of the Requisite Variety Principle [9]. One unit may be thought of as the systems, while another unit can be thought of as an element of the ecosystem with which structure must interface. When two units of similar intricacy, i.e., with the same range of behaviors, but one functioning on a greater scale, e.g., two extremely tightly regulated militaries, but one military bigger than another, the relatively large military will most likely prevail. The generally higher military will probably win when two militaries of equatable scale but inequitable variability (- for example, two evenly large and supercharged squadrons, but just one being much more versatile than others), because the significantly greater military has had an intervention with every intervention of the relatively low military but not conversely. When a relatively small military, such as a guerrilla force, clashes with a larger- 
scale military, such as the American military in Vietnamese or the Russian army in Tajikistan, focus is on the terrain. The military with greater complexity at bigger sizes is preferred in wide fields and open seas, while greater intricacy at local scale is preferred in the jungle and highlands.

Medicine, for instance, entails both small-scale activities with relatively high intricacy, like crisis intervention, and large-scale, lesser tasks, such as vaccine production and delivery. Because virtually every patient experiences the identical activities, vaccinations have a lesser level of complexity but a bigger size. Large-scale leading companies and initiatives are well-suited to large-scale, low-complexity activities, while crisis intervention necessitates healthcare systems with a significant level of comparatively tiny (i.e., localized) intricacy [10].

A multi-resolution complexities discrepancy may be seen in the eurozone. Fiscal policy is primarily made at the national level, resulting in greater complexity at the federal level but comparatively little complex nature at the eurozone level, whereas monetary policy is primarily made at the eurozone level, resulting in some ambiguity at the eurozone level but an incapability to vary (i.e., complexity) at the national level. Many believe that the eurozone's economic problems are the result of this misalignment, which prevents efficient monetary and fiscal policy interactions [11].

Problems occur from incompatibilities between intricacies of a job to be done and the intricacies of a system executing that work, rather than from an excess of or a lack of complexity (at any scale). (Incidentally, human emotions seem to mirror this principle: we get bored when our surroundings are too basic, and overwhelmed when they are too complicated.) It's worth noting that the system inside one situation may be the assignment in the other; for example, the same intricacy that allows a system to engage with its environment could hinder other systems from effectively managing it. The complexity profiles and scales have not been computed or specified exactly in any of the examples above. Rather, size proxies are employed, and complexity comparisons are calculated. Although such a method cannot provide exact findings (indeed, no technique can, considering the complexity that a clear understanding of such networks would need), further accuracy is not required when even a rough study shows significant complexity incompatibilities. (Detailed analysis may be needed to correct the identified mismatches.) Although it may be easy to blame issues emerging from a complexity imbalance on certain causative factors and sequences of events, difficulties will continue to arise in some form or another until the fundamental disparity is resolved.

\section{Split Structures}

There is still the potential of a complexity mismatch, even if the system's complexity matches that of its surroundings at the proper scales. Imagine 2 kinds of friends - a total of four individuals who can each carry 100 pounds - and two 200 pounds sofas that must be transported. Assume that each individual can coordinate with one of her friends but not with the other two. Since each couple of friends can raise one of 200-pound couches, the overall complexity of the human system is adequate to move all couches at the proper scales [12]. If one person from every pair of friends was assigned to each sofa, they would be unable to raise the couches since the two individuals lifting every sofa would not be from the same pairing of buddies and therefore will not be responsible for coordinating their movements. The issue is that, although the pairs of friends have enough complexities at the appropriate scales to lift the sofas, the subdivisions within the network of friends do not correspond to the natural subdivisions inside the systems of couches. When we concentrate on just one sofa, we can observe the mismatch in complexity: although the couch needs concrete effort on the order of 200 pounds, the two individuals lifting it are only capable of two separate acts on the order of 100 pounds each.

The organizational structure of academic departments offers a more comparable example of subdivision incompatibility. Academic institutions have various layers of division (agencies, subspecialties, etc.) to arrange expertise and coordinate people, leading to a high overarching complexity across various scales, where scale refers to either the multitude of synchronised individuals or the quantity of synchronised expertise, depending about which component of the education system is being considered [13]. Conversely, the collection of issues that academics may possibly address has many degrees of natural subgroup, with each subdivision needing different kinds of organized understanding and effort to solve. Academia's multi-scale complexity enables it to tackle many of these issues successfully. Nevertheless, even if there is adequate overall multiscale intricacy, there may be issues that academy is unable to address because the divisions inside the dilemma do not meet the divisions within academy. Over the last several decades, the number of multidisciplinary institutes and initiatives has risen, indicating that such a mismatch is seen. However, the academic institution as a whole may continue to stymie progress on issues that do not neatly fit into a field or subdiscipline.

The preceding examples demonstrate the concept that in sequence for a system to preferentially react to an array of external behavioural patterns, not only must the framework here seem to have at least quite so much intricacies at all measurements as this array of external behavioural patterns, but each subsection of the scheme must also have at least quite so much sophistication at any and all scales as the ecologic behaviour patterns correlating to that subcategory [14]. A reasonable rule of thumb is that choices about independent elements or features of a system ought to be able make separately, whereas decisions about dependent elements of the project must be made dependently. As a result, organisations that make such choices should be split in the same way that inherent boundaries in the ecosystems in which they interact are divided. The partitions in the human brain, as well as the study of divisions in neuronal networks in general, show that subsystems that are partitioned to reflect the inherent divisions in their surroundings surpass systems with greater internal connection. Section III below focuses of complex systems and their uncertainties.

\section{COMPLEX STRUCTURES (CS) AND UNCERTAINTIES}

Despite the fact that the concepts outlined in the preceding chapter identify the underlying qualities and restrictions of organizations, our recognition of most complicated systems will always be flawed. And, no matter how well-thought-out a 
design is, a genuinely sophisticated network will introduce components that were not anticipated. It's also worth noting that in a complicated functioning system, the possible positive effect of a modification is usually considerably lower than the possible bad impact. A little adjustment in a computer's circuits, for instance, is unlikely to increase the computer's speed significantly, but it could force it to collapse. Other case is aircraft. This is due to the fact that a significant level of intricacy entails that many structure designs will not function for every one that will.

How can we be sure that structures we build or are a part of will succeed in the lack of total understanding? Whereas many structures rely on the concept that excellent judgements will be made, certain structures do not rely on personal intelligence and can work effectively even when decision-makers are fallible (whether due to corruption, subconscious bias, or the fundamental limitations of human minds). The analysis of sophisticated structures takes a systematic technique to this finding by viewing decision-makers as members of the structure with restricted intricacy and decision-making capability (implicit or explicit). As a result, the challenge becomes: how can we create structures that are more sophisticated than the decision-makers who operate them?

\section{Evolutionary Procedures}

Whereas most structures are weakened by ambiguity and complexity, other structures actually gain from it. The implementation of some form of evolutionary change, in which effective modifications are replicated (and further refined) but negative modifications are not, is a frequent trait of these structures. The classical evolution mechanisms are genetic: animals with the scale and complexity of humans developed from single-celled creatures as a result of diversity brought by genetic changes. Individuals, in addition, have the ability to prosper from unpredictable disturbances (provided the shocks are not too strong). Prior intervention to nonlethal infections improves immune response functioning; microtears and microfractures enhance bones and muscles, accordingly; we learn through new knowledge and problem-solving; and our behavioral patterns are reinforced by challenge, providing the challenges are not too extreme.

Another illustration of how structures can survive in the face of uncertainties is aggressive economic systems. Due to our inability to predict which ideas and firms will prosper, a large number of possible inventions and enterprises must be developed and enhanced simultaneously, with the profitable ones increasing and the average ones collapsing. The effectiveness of these can then be increased in the same way, with multiple ways being used at the same time, and so on. (Nevertheless, large- scale components of the market structure may maximize for the wrong purposes, stabilizing into damaging society stability in the absence of adequately managed multiresolution coordinated structures.)

Similarly, huge companies' internal systems may adopt an evolving trend in which little sections of the corporation collapse and are therefore reformed; without such adaptability, the whole company may fail all at once in response to a change internally or externally. In some circumstances, the collapse of an entire enterprise allows for the emergence of more successful organisations (provided that the economic is suitably fragmented and competition and that the company in consideration is not "too large to collapse"). Nonetheless, because the fall of democracy is rarely one of these scenarios, it is critical that management structures have the ability to gain institutionally from randomization and unpredictability. Contrary to popular belief, preventing tiny breakdowns from occurring can degrade systems over time by slowing evolution process and establishing interrelations that might lead to systemic danger.

In attempt to succeed in the face of uncertainties and to outperform the intricacy of personal judgment, structures can contain evolutionary mechanisms, which will allow them to develop over duration, even if they are initially constrained [15]. The first stage is to give the structure enough variance so that it can examine the range of alternatives. Because a lot of variance involves a lot of intricacy, and sophistication exchanges off with scale, this kind of variability has to happen at small scale (in both space and time). For instance, allowing each town to explore individually in the area of government enables for several strategies to be tested out in concurrently and repeated on. The alternative method would be to implement a single government strategy, the consequences of which would be impossible to compare.

The second phase is to enable communication among different sections of the structure so that good decisions can be replicated and expanded upon somewhere else (e.g., cities copying the successful practices of other cities). Unfortunate consequences will always occur; the goal is to allow unplanned outcomes to benefit instead of harm the structure as a whole. Because an explicitly architecture is a behaviour of the first mechanism, structures can only clearly construct platforms of lower intricacy. Structures that develop over time, on the other hand, can be more intricate than their architects. To enable intricacy to expand spontaneously over time, the urge for absolute authority must be abandoned.

\section{Multiscale Evolutionary Procedures}

Effective evolution systems, on the whole, do not include uncontrolled struggle, but rather include both competitivee market and collaboration at many levels. Cellular collaborate inside complex creatures to better compete with other creatures, and individuals collaborate both within and between organisms to better compete with other individuals. Since an organization must cooperate within itself in order to efficiently contend with another organization (huge competitiveness), competitive rivalry at a bigger scale inevitably generates collaboration at a smaller level.

Collaboration may promote competitiveness, because the greatest way for an organization to accomplish its common objectives is to foster genuine competition among its divisions. To compete fairly with one another, those divisions must develop collaboration within themself, and they, too, may be capable to improve the efficiency of their interior coordination by establishing some constructive rivalry among their individuals. If these individuals form organizations, the cycle of rivalry leading to collaboration, which leads to greater rivalry, can be repeated on a lower scale. This mechanism can also happen in reverse: in attempt to operate more successfully, individual may collaborate with one another to join 
alliances, which in return may collaborate to build even bigger numbers, and so on. As a result, a sophisticated system of cooperative and rivalry between organizations of different proportions (scales) can emerge spontaneously.

\section{CONCLUSION}

Spontaneous large-scale responses of structures with numerous components may frequently be deduced from the actions of their individual components. Despite the difficulty of explaining the specifics of trillions of particles, statistical physics discovered that the macroscopic characteristics of molecules may be well comprehended by examining their range of potential behaviors rather than their particular configurations and movements. Whereas many macroeconomic characteristics may be explained in terms of having molecular behaviors, some physical processes, such as phase transformations, cannot be comprehended by averaging across system components; as a result, physicists have been compelled to create new, multiscale techniques. Similarly, although traditional statistical methods-which deduce the average characteristics of a system's numerous components - can effectively represent certain social and biological networks, they fail miserably in others. Adopting a systemic approach and examining the range of potential actions may provide knowledge that cannot be gained by focusing only on the causes and consequences of specific issues or crises. In almost the same manner that volatility is the beginning point for statistical physics, a system's complexity, which is determined by the number of different possible behaviors (i.e., the space of possibilities), is a starting point for determining its large-scale characteristics. Because a system's number of unique behaviors is proportional to its degree of detail (behaviour patterns that seem to be the same at smaller screen may be distinctive at greater resolution), complexity is scale dependent. Interconnections between elements decrease complexity at lower sizes by limiting individual elements freedom, while they increase complexity at higher scales by allowing behaviors that require many components cooperating. Thus, there is a basic tradeoff between both the variety of behaviours at smaller and bigger sizes for systems made up of the same elements. This scale tradeoff is linked to the balance between a system's adaptability, which is determined by the diversity of reactions it has to adverse shocks, as well as its economy, which is determined by its operational size. There is no optimum scale for a system's intricacy; instead, the most account for the majority are those that match the sophistication of their surroundings at every scale.

\section{References}

[1]. A. Rizzo, "Guest Editorial From Complex Systems Science to Complex Systems Technology and Return: A Tale of an Endless Loop", IEEE Systems Journal, vol. 6, no. 3, pp. 479-480, 2012. Doi: 10.1109/jsyst.2012.2212776.

[2]. G. Mobus, "A Framework for Understanding and Achieving Sustainability of Complex Systems", Systems Research and Behavioral Science, vol. 34, no. 5, pp. 544-552, 2017. Doi: 10.1002/sres.2482.

[3]. J. Holland, "Studying Complex Adaptive Systems", Journal of Systems Science and Complexity, vol. 19, no. 1, pp. 1-8, 2006. Doi: 10.1007/s11424-006-0001-z.

[4]. S. Banerjee, "Citizen Data Science for Social Good in Complex Systems: Case Studies and Vignettes from Recent Projects", Interdisciplinary Description of Complex Systems, vol. 16, no. 1, pp. 88-91, 2018. Doi: 10.7906/indecs.16.1.6.

[5]. J. Zhang, "Complexity and Universality of Iterated Finite Automata", Complex Systems, vol. 18, no. 1, pp. 145-158, 2009. Doi: 10.25088/complexsystems.18.1.145.

[6]. I. Macaluso, C. Galiotto, N. Marchetti and L. Doyle, "A complex systems science perspective on wireless networks", Journal of Systems Science and Complexity, vol. 29, no. 4, pp. 1034-1056, 2016. Doi: 10.1007/s11424-016-4122-8.

[7]. J. Zhang, "Energy flows in complex ecological systems: a review", Journal of Systems Science and Complexity, vol. 22, no. 3, pp. 345-359, 2009. Doi: $10.1007 / \mathrm{s} 11424-009-9169-3$.

[8]. B. Johnson and A. Hernandez, "Exploring Engineered Complex Adaptive Systems of Systems", Procedia Computer Science, vol. 95, pp. 5865, 2016. Doi: 10.1016/j.procs.2016.09.293.

[9]. B. Markey-Towler, "Modelling Behavioural Systems: Integrating Psychology and Complex Systems Science", SSRN Electronic Journal, 2020. Doi: $10.2139 / \mathrm{ssrn} .3519804$.

[10]. C. Adler and C. Dagli, "Enabling Systems and the Adaptability of Complex Systems-of- Systems", Procedia Computer Science, vol. 12, pp. 31-36, 2012. Doi: 10.1016/j.procs.2012.09.025.

[11]. H. Karimi, "Complex Engineering Systems", Complex Engineering Systems, 2021. Doi: 10.20517/ces.2021.01.

[12]. V. Panfilov, "Complex Technological Systems In Agroindustrial Complex Development", Vestnik of the Russian agricultural science, no. 1, pp. 13-16, 1970. Doi: 10.30850/vrsn/2019/1/13-16.

[13]. R. Foote, "Mathematics and Complex Systems", Science, vol. 318, no. 5849, pp. 410-412, 2007. Doi: 10.1126/science.1141754.

[14]. K. NORWICH, "The tactile discrimination of complex systems", International Journal of Systems Science, vol. 3, no. 2, pp. 225-228, 1972. Doi: $10.1080 / 00207727208920262$.

[15]. L. Beckerman, "Application of complex systems science to systems engineering", Systems Engineering, vol. 3, no. 2, pp. 96-102, 2000. Doi 10.1002/1520-6858(2000)3:2<96::aid-sys4>3.0.co;2-7. 